CPP-95-10

DOE-ER-40757-067

July 1995

\title{
Four Top Production and Electroweak Symmetry Breaking
}

\author{
Kingman Cheung \\ Center for Particle Physics, University of Texas at Austin, Austin TX 78712
}

\begin{abstract}
With the recent discovery of a heavy top quark $\left(m_{t} \approx 175-200 \mathrm{GeV}\right)$, the top quark opens an window to electroweak symmetry breaking. We propose the study of fourtop, $t \bar{t} t \bar{t}$, production at hadronic supercolliders as a probe to electroweak symmetry breaking.
\end{abstract}

Recent results of the top-quark search by CDF [1] and D0 [2] at the Tevatron showed the existence of the sixth quark - the top quark. The measured mass was $176 \pm 8 \pm 10 \mathrm{GeV}$ from CDF and $199_{-21}^{+19} \pm 22 \mathrm{GeV}$ from D0. The standard model (SM) [3] that was proposed more than 20 years ago is confirmed to a solid ground as all the ingredients were found, except for electroweak symmetry breaking (EWSB). The understanding of EWSB is necessary to explain all fermion and gauge boson masses on a fundamental level. The goal of the next generation of colliders is the exploration of the EWSB sector. The dynamics of the EWSB sector can be probed by studying longitudinal vector boson scattering [4], and also through the indirect implication from precision measurements [5].

Since the top quark is very heavy it provides another avenue to study EWSB because the coupling of the top to the Goldstone bosons and the Higgs boson is of order $\frac{g m_{t}}{2 m_{W}}=$ $0.7\left(m_{t} / 175 \mathrm{GeV}\right) \sim \mathcal{O}(1)$. Therefore, the studies of the interactions among the top quark, goldstone bosons, and the EWSB sector enable one to reach the nonperturbative regime of the sector. Thus, their interactions can give light to the structure of the EWSB sector. As a remark here, the production of $g g, q \bar{q} \rightarrow t \bar{t}$ has been proposed as a probe to extended color 
sectors beyond $\mathrm{SM}$, e.g., $\mathrm{SU}(3)_{L} \times \mathrm{SU}(3)_{R} \supset \mathrm{SU}(3)_{C}$ [6], to some technicolor resonances [7], or to the top-color models [8]. Since we are interested only in EWSB, we assume that there is no complicated color sectors other than the usual $\mathrm{SU}(3)_{C}$, and the light fermions and gluons do not couple directly to the new physics of EWSB. Under our assumptions the top production via $g g, q \bar{q} \rightarrow t \bar{t}$ will not deviate from the SM, except for the $t \bar{t}$ rescattering via the new interactions of the EWSB sector. This is certainly an interesting area to study. For example, the Higgs boson can contribute to the $t \bar{t}$ production via $g g \rightarrow H \rightarrow t \bar{t}$, which interferes nontrivially with $g g \rightarrow t \bar{t}$ to give interesting structures near the resonance peak in the $m_{t \bar{t}}$ spectrum [9]. But due to enormous QCD $g g \rightarrow t \bar{t}$ production and the other decay channels of the Higgs boson, it is difficult to detect the nontrivial structures around the resonance peak [9].

In this letter we propose the study of four top, $t \bar{t} t \bar{t}$, production as a probe to EWSB. This reaction is more advantageous than just the $t \bar{t}$ production when the new physics does not couple to light quarks or gluons. Another reason is that since the four-top production is actually small at the LHC energies such that any appreciable enhancement from the SM predictions is easy to detect. The schematic diagram for the signal of $t \bar{t} t \bar{t}$ production via EWSB interactions is shown in Fig. 1, in which the tops and antitops come from the splitting of gluons. Two of the tops are strongly scattered via the new interactions of the EWSB sector, and the other two simply go off without interacting. We refer the latter as spectator tops. In the following we shall study this signal using the effective Lagrangian method, and by specifying some particular resonances. We shall show that in the effective Lagrangian approach the enhancement of cross sections, due to the 4-fermion operators, is substantial. On the other hand, SM Higgs boson can only give a marginal enhancement over the background. But for the $\mathrm{TeV}$ vector resonances, the cross sections could be very large because of absence of bounds on the coupling. Finally, we show that this reaction is feasible as a probe to EWSB, even under the presence of QCD backgrounds.

We first use the effective Lagrangian approach to parameterize the scattering part of the 
reaction, $t \bar{t} \rightarrow t \bar{t}$. Assuming all heavy particles in the underlying theory have been integrated out, $t \bar{t} \rightarrow t \bar{t}$ interactions can be parameterized by a set of dimension-six 4 -fermion operators $\left\{O_{i}\right\}$ as

$$
\mathcal{L}_{\text {int }}=\sum_{i} \frac{C_{i}}{\Lambda^{2}} O_{i},
$$

where $C_{i}$ 's are the coefficients of the operators, and $\Lambda$ is the cut-off scale, below which the effective theory is valid. The operators are

$$
\begin{aligned}
O_{0}=(\bar{t} t)(\bar{t} t), & O_{0}^{\prime}=\left(\bar{t} T^{a} t\right)\left(\bar{t} T^{a} t\right) \\
O_{1}=\left(\bar{t} \gamma_{5} t\right)\left(\bar{t} \gamma_{5} t\right), & O_{1}^{\prime}=\left(\bar{t} \gamma_{5} T^{a} t\right)\left(\bar{t} \gamma_{5} T^{a} t\right) \\
O_{2}=\frac{1}{2}\left(\bar{t} \gamma_{\mu} t\right)\left(\bar{t} \gamma^{\mu} t\right), & O_{2}^{\prime}=\frac{1}{2}\left(\bar{t} \gamma_{\mu} T^{a} t\right)\left(\bar{t} \gamma^{\mu} T^{a} t\right) \\
O_{3}=\frac{1}{2}\left(\bar{t} \gamma_{\mu} \gamma_{5} t\right)\left(\bar{t} \gamma^{\mu} \gamma_{5} t\right), & O_{3}^{\prime}=\frac{1}{2}\left(\bar{t} \gamma_{\mu} \gamma_{5} T^{a} t\right)\left(\bar{t} \gamma^{\mu} \gamma_{5} T^{a} t\right)
\end{aligned}
$$

where $T^{a}$ are the $\mathrm{SU}(3)_{C}$ color matrices. Operators of higher dimensions are suppressed by extra powers of $\Lambda$. Each operator should correspond to the dynamics of the underlying theory, e.g., the operator $O_{3}$ arises from an exchange of technirho $\left(\rho_{T C}\right)$ of technicolor theories. A few remarks regarding $C_{i}$ 's and $\Lambda$ are in order. (i) The size of the coefficients $C_{i}$ 's, by naive dimension analysis, is of order but less than $16 \pi^{2}$. The coefficient, say, $C_{0}$ can be estimated from the heavy Higgs model, $m_{H}=1 \mathrm{TeV}$, by integrating out the heavy Higgs boson in the $t \bar{t} \rightarrow t \bar{t}$ scattering. Since $\mathcal{L}_{t t H}=g m_{t} /\left(2 m_{W}\right) t t H$, the effective four fermion coupling is

$$
\frac{g^{2} m_{t}^{2}}{4 m_{W}^{2}} \frac{1}{m_{H}^{2}} \approx \frac{0.5}{m_{H}^{2}}
$$

for $m_{t}=175 \mathrm{GeV}$. Therefore, $C_{0}$ is of order 1 if $\Lambda=1 \mathrm{TeV}$ in the heavy Higgs model. We also expect other $C_{i}$ 's to be of order 1. (ii) We expect the new physics of EWSB to come in in the order of $\mathrm{TeV}$, we therefore choose $\Lambda=1 \mathrm{TeV}$. Results for other choices of $\Lambda$ can be easily implied. (iii) We do not consider the interference between the $\mathcal{L}_{\text {int }}$ of Eq. (1) and the SM. The leading SM $t \bar{t} \rightarrow t \bar{t}$ scattering is via a single gluon exchange, so that it does not have interference with the operators $\left(O_{0}-O_{3}\right)$, which are color-singlet. The primed operators $\left(O_{0}^{\prime}-O_{3}^{\prime}\right)$ actually interfere with the single gluon-exchange $t \bar{t} \rightarrow t \bar{t}$ diagrams, but they give much smaller results than the unprimed ones (see results below). Therefore, the 
scattering amplitude, due to the new interactions, scales as $C_{i} / \Lambda^{2}$, and the resulting cross sections as $C_{i}^{2} / \Lambda^{4}$. In addition, we only look at one operator at a time. Later, we shall give the signal cross sections in the form of $\sum_{i} \frac{C_{i}^{2}}{\Lambda^{4}} a_{i}$, where $a_{i}$ 's are calculated by $t \bar{t}$ scattering.

Next, we consider the specific dynamics of the underlying theory. The simplest model is the SM with one Higgs doublet. The coupling of the SM Higgs boson to the top quark is given by $\mathcal{L}_{t t H}=\frac{g m_{t}}{2 m_{W}} \bar{t} t H$. In principle, we can also consider a model with nonstandard Yukawa coupling

$$
\mathcal{L}_{t t h}=\bar{t}\left(a+i b \gamma_{5}\right) t h
$$

where $a, b$ are real constants. The width of $h$ into $t \bar{t}$ is given by

$$
\Gamma_{h \rightarrow t \bar{t}}=\frac{3 m_{h}}{8 \pi}\left[a^{2}\left(1-\frac{4 m_{t}^{2}}{m_{h}^{2}}\right)+b^{2}\right]\left[1-\frac{4 m_{t}^{2}}{m_{h}^{2}}\right]^{1 / 2}
$$

The spin- and color-averaged amplitude squared for $t \bar{t} \rightarrow t \bar{t}$ via the Higgs boson $h$ of Eq. (四) is

$$
\begin{aligned}
& \sum|\mathcal{M}|^{2}=\frac{1}{9} \frac{1}{4}\left\{\frac{36\left[s\left(a^{2}+b^{2}\right)-4 a^{2} m_{t}^{2}\right]^{2}}{\left(s-m_{h}^{2}\right)^{2}+\Gamma_{h}^{2} m_{h}^{2}}+\frac{36\left[t\left(a^{2}+b^{2}\right)-4 a^{2} m_{t}^{2}\right]^{2}}{\left(t-m_{h}^{2}\right)^{2}}-6 \times\right. \\
& \left.\frac{8 m_{t}^{2}\left(2 m_{t}^{2}\left(a^{4}-2 a^{2} b^{2}-b^{4}\right)+u\left(a^{2}+b^{2}\right)^{2}-a^{2}\left(a^{2}+b^{2}\right)(s+t)\right)+\left(a^{2}+b^{2}\right)^{2}\left(s^{2}+t^{2}-u^{2}\right)}{\left(t-m_{h}^{2}\right)\left[\left(s-m_{h}^{2}\right)^{2}+\Gamma_{h}^{2} m_{h}^{2}\right]^{\frac{1}{2}}}\right\}
\end{aligned}
$$

where we used the Breit-Wigner prescription for the $s$-channel Higgs propagator, and we chose the width $\Gamma_{h}$ to be the same as the SM one, $\Gamma_{H}\left(m_{H}=1 \mathrm{TeV}\right) \approx 0.5 \mathrm{TeV}$. The SM results can be recovered by putting $a=g m_{t} /\left(2 m_{W}\right)$ and $b=0$.

As mentioned above the operator $\mathrm{O}_{3}$ can be induced by a technirho. Drawing the analogy with the QCD interaction of $\rho$ to nucleons, we can write down the interaction of the $\rho_{\mathrm{TC}}$ with the top quarks [10]

$$
\mathcal{L}_{t t \rho_{\mathrm{TC}}}=g_{t t \rho_{\mathrm{TC}}}\left(\bar{t} \gamma_{\mu} t\right) \rho_{\mathrm{TC}}^{o \mu}
$$

Similar expression is for the $\omega_{\mathrm{TC}}$. Since $\rho(770)$ and $\omega(783)$ decay primarily into pions, the $\rho_{\mathrm{TC}}$ and $\omega_{\mathrm{TC}}$ will also decay primarily into the Goldstone bosons, which are the longitudinal 
component of the $W$ and $Z$ bosons. Therefore, we expect that the decay of $\rho_{\mathrm{TC}}$ and $\omega_{\mathrm{TC}}$ into $t \bar{t}$ are relatively rare. In fact, the coupling is given in Ref. [10]

$$
\mathcal{L}_{t t \rho_{\mathrm{TC}}}=\frac{f_{\rho_{\mathrm{TC}}} v^{2}}{2 f^{2}} \rho_{\mathrm{TC}}^{o \mu}\left(\bar{t} \gamma_{\mu} t\right)
$$

where $f \approx \mathcal{O}(1 \mathrm{TeV}), f_{\rho_{\mathrm{TC}}} \approx f_{\rho}\left(3 / N_{\mathrm{TC}}\right)^{1 / 2}$, and $v \approx 246 \mathrm{GeV}$. Using $N_{\mathrm{TC}}=4, f_{\rho}=5.7$, and $f=1 \mathrm{TeV}$, we find $g_{t t \rho_{\mathrm{TC}}}=f_{\rho_{\mathrm{TC}}} v^{2} /\left(2 f^{2}\right) \approx 0.15$, while the coupling constant $g_{t t H}=$ $g m_{t} /\left(2 m_{W}\right)$ is about 0.71 . This already tells us that $t \bar{t} \rightarrow t \bar{t}$ scattering cross section via $\rho_{\mathrm{TC}}$ is down by a factor of $(0.71 / 0.15)^{4} \approx 500$, compared to the cross section via the SM Higgs boson. In the following we do not confine to Technicolor models but assume a general massive vector boson $V^{\mu}$ of $\mathrm{TeV}$ mass and the coupling constant to be of order 1, i.e.,

$$
\mathcal{L}_{t t V}=g_{t t V}\left(\bar{t} \gamma_{\mu} t\right) V^{\mu}
$$

The decay width of $V^{\mu} \rightarrow t \bar{t}$ is given by

$$
\Gamma_{V \rightarrow t \bar{t}}=\frac{g_{t t V}^{2}}{4 \pi} m_{V} \quad \text { for } m_{V} \gg m_{t}
$$

and $V^{\mu}$ is $\mathrm{SU}(3)_{C}$ color singlet. The spin- and color-averaged amplitude squared for $t \bar{t} \rightarrow t \bar{t}$ via the vector field $V^{\mu}$ is given by

$$
\begin{aligned}
\bar{\sum}|\mathcal{M}|^{2} & =\frac{1}{9} \frac{1}{4} g_{t t V}^{4}\left\{\frac{72\left[4 m_{t}^{2}\left(2 m_{t}^{2}+s-t-u\right)+t^{2}+u^{2}\right]}{\left(s-m_{V}^{2}\right)^{2}+\Gamma_{V}^{2} m_{V}^{2}}\right. \\
& \left.+\frac{72\left[4 m_{t}^{2}\left(2 m_{t}^{2}-s+t-u\right)+u^{2}+s^{2}\right]}{\left(t-m_{V}^{2}\right)^{2}}+\frac{48\left[2 m_{t}^{2}\left(2 m_{t}^{2}+s+t-3 u\right)+u^{2}\right]}{\left(t-m_{V}^{2}\right)\left[\left(s-m_{V}^{2}\right)^{2}+\Gamma_{V}^{2} m_{V}^{2}\right]^{\frac{1}{2}}}\right\}
\end{aligned}
$$

The total width $\Gamma_{V}$ depends on $m_{V}, g_{t t V}$, and the couplings to gauge bosons. We assume that the decay width of $V^{\mu}$ into gauge bosons are much larger than that into fermions. Specifically, we chose the total width $\Gamma_{V}$ to be $5 \Gamma_{V \rightarrow t \bar{t}}$. For $g_{t t V} \approx 1$ and $m_{V}=1 \mathrm{TeV}$, the width $\Gamma_{V \rightarrow t \bar{t}} \approx 80 \mathrm{GeV}$ and the total width $\Gamma_{V}=400 \mathrm{GeV}$. This model is, therefore, specified by any two of these three parameters: $m_{V}, \Gamma_{V}$, and $g_{t t V}$.

The subprocess cross section is folded with the effective luminosity for the top quark inside the proton. The top as a parton inside the proton can be regarded as originating from 
gluon splitting

$$
\mu \frac{\partial}{\partial \mu} f_{t}(x, \mu)=\int_{x}^{1} \frac{d y}{y} f_{g}(y, \mu) P_{g \rightarrow t}\left(\frac{x}{y}, \mu\right),
$$

where $f_{g}(x)$ is the gluon parton distribution function and $P_{g \rightarrow t}(x)$ is the Altarelli-Parisi splitting kernel. This equation gives only the leading order results. We, instead, employ the top-parton distribution of the most recent CTEQ (v.3) [11] structure functions.

First, we show the results using the effective Lagrangian method. At the LHC energy $(14 \mathrm{TeV})$ and $m_{t}=175 \mathrm{GeV}$, we get the $t \bar{t} t \bar{t}$ cross section in fb:

$$
\begin{aligned}
\sigma_{p p}(t \bar{t} t \bar{t})= & \frac{1}{\Lambda^{4}[\mathrm{TeV}]}\left(C_{0}^{2}(2.04)+C_{1}^{2}(2.20)+C_{2}^{2}(2.09)+C_{3}^{2}(1.96)\right. \\
& \left.+C^{\prime 2}(0.57)+C^{\prime 2}{ }_{1}^{2}(0.62)+{C^{\prime}}_{2}^{2}(0.36)+C^{\prime 2}(0.34)\right) .
\end{aligned}
$$

As we shall see below these cross sections are substantially larger than the background for a moderate choice of $C_{i}$ 's. Next, the SM Higgs boson gives only a small cross section of $0.7-1.2 \mathrm{fb}$ for $m_{t} \approx 175-200 \mathrm{GeV}$. For the general Higgs model of Eq. (雨) we chose the coupling constant $a$ to be the same as the SM value, $\frac{g m_{t}}{2 m_{W}}$, and $b=a / 5$. It gives only about $10 \%$ larger cross sections than the SM Higgs boson. On the other hand, the vector resonance model gives potentially much larger cross sections. We chose $m_{V}=1 \mathrm{TeV}$ and the total width $\Gamma_{V}$ to be $0.3(0.5) \mathrm{TeV}$. According to our assumption, $\Gamma_{V \rightarrow t \bar{t}}=\Gamma_{V} / 5$, and Eq. (10), we have $g_{t t V}=0.87(1.12)$. The resulting cross section is $5.8(9.4) \mathrm{fb}$.

So far, we have not considered backgrounds. The major background is the QCD production of four top, $t \bar{t} t \bar{t}$, at the order of $\alpha_{s}^{4}$ via the processes $g g, q \bar{q} \rightarrow t \bar{t} t \bar{t}$ [12]. Among these two processes, $g g \rightarrow t \bar{t} t \bar{t}$ is the dominant one at the LHC energies and it is closer to the event topology of the signal than the other one. We verified that the cross section for $q \bar{q} \rightarrow t \bar{t} t \bar{t}$ is only $10-15 \%$ of that for $g g \rightarrow t \bar{t} t \bar{t}$ with or without the cuts that we apply below.

In the above calculations, the kinematics of the spectator tops cannot be obtained by the method of effective top-parton luminosity. We need an exact calculation of the signal to compare with the background. Because the kinematics of the spectator tops is independent of the interaction models of the scattered tops, we can look at one particular model that can 
be calculated exactly, and the kinematics thus obtained is also valid for other models. The SM Higgs model can be calculated exactly as $g g \rightarrow t \bar{t} H$ followed by $H \rightarrow t \bar{t}$. Therefore, in the following we shall distinguish the signal, $g g \rightarrow t \bar{t} H \rightarrow t \bar{t} t \bar{t}$, from the QCD background, $g g \rightarrow t \bar{t} t \bar{t}$, by comparing the distributions of a few observables.

We start with the cuts for observing the tops

$$
p_{T}(\text { top })>20 \mathrm{GeV} \quad \text { and } \quad \mid y(\text { top }) \mid<5 .
$$

There is a technical problem in identifying which are the spectator tops and scattered tops. One solution is to order the tops according to their transverse momenta as

$$
p_{T}\left(t_{1}\right)<p_{T}\left(t_{2}\right)<p_{T}\left(t_{3}\right)<p_{T}\left(t_{4}\right),
$$

in which we identify the tops with smaller $p_{T}\left(t_{1}\right.$ and $\left.t_{2}\right)$ as the spectator tops, and the tops with larger $p_{T}\left(t_{3}\right.$ and $\left.t_{4}\right)$ as the scattered tops. We verified that this method of identifying the spectator and scattered tops is very close to the theoretical case when distributions are concerned. Once we identify the tops, we can impose a large invariant mass cut on the scattered pair, since they are scattered via the new strong interaction of EWSB, while the tops from the background interact only via the QCD interaction, which becomes weak at the TeV scale. Specifically, we impose

$$
m_{t \bar{t}}>600 \mathrm{GeV}
$$

since it will not hurt the signal for, e.g., a $1 \mathrm{TeV}$ resonance with a width of a few hundred GeV. Among many observables, $\Delta p_{T}\left(t_{3}, t_{4}\right)$ and $y_{\text {gap }}\left(t_{1}, t_{2}\right)$ are useful. $\Delta p_{T}\left(t_{3}, t_{4}\right)$ is the vector difference between the transverse momenta of the scattered tops:

$$
\Delta p_{T}\left(t_{3}, t_{4}\right)=\left|\vec{p}_{T}\left(t_{3}\right)-\vec{p}_{T}\left(t_{4}\right)\right| .
$$

This observable is used to exploit the back-to-back nature of the scattered tops in the transverse plane after the strong scattering. The normalized differential cross sections versus $\Delta p_{T}\left(t_{3}, t_{4}\right)$ are shown in Fig. 2(a). From Fig. 2(a) a further cut of, say, $\Delta p_{T}\left(t_{3}, t_{4}\right)>600$ 
$\mathrm{GeV}$ can improve the signal. The observable $y_{\text {gap }}$ is the difference in rapidities of the spectator tops, given by

$$
y_{\text {gap }}=\left|y\left(t_{1}\right)-y\left(t_{2}\right)\right| .
$$

We show the normalized differential cross section versus $y_{\text {gap }}$ in Fig. 目(b). We observe a dip in the region $y_{\text {gap }} \lesssim 1.2$ for the signal but not for the background. This is easily explained by the color-flow between the spectator tops, analogous to the rapidity gap physics [13]. When the central tops are scattered by a colorless bosons (in this case the SM Higgs), there is no net color-flow between the two spectator tops. Therefore, we expect a rapidity gap to exist between the two spectator tops in the signal, while it is not true for the background. It is particularly interesting for the colorless models, since from Eq. (13) we notice that the cross sections are much larger when the tops are scattered by colorless bosons than by colored bosons.

We give the effectiveness of the various cuts in Table @. If the signal and background start with the same cross section before the cuts, the signal will end up 5 times as large as the background after all the cuts, as shown in Table [. The starting cross section for the background process $g g \rightarrow t \bar{t} t \bar{t}$ ranges from 3 to $7 \mathrm{fb}$ for the factorization scale being varied from $\sqrt{\hat{s}}$ to $\sqrt{\hat{s}} / 4$. Therefore, the background, after all the cuts, ranges between 0.3 to 0.7 $\mathrm{fb}$. On the other hand, before the cuts the smallest signal is $0.7-1.2 \mathrm{fb}$ coming from the SM Higgs boson and the largest is 5.8(9.4) fb from the vector resonance of mass $1 \mathrm{TeV}$ and width $0.3(0.5) \mathrm{TeV}$. Therefore, after applying the cuts in Table $\mathbb{\text { a }}$, there is a large excess of the signal for the vector resonance $(2.8-4.6 \mathrm{fb})$ and for the effective Lagrangian method over the background (0.3-0.7 fb), but only a marginal excess for the SM Higgs signal (0.3-0.6 fb).

We also emphasize the importance of the invariant mass $m_{t \bar{t}}$ distribution, which is useful in identifying any resonance structures or enhancement in the $\mathrm{TeV}$ region. So far, we only presented results for $m_{t}=175 \mathrm{GeV}$. The feasibility of using $t \bar{t} t \bar{t}$ production to probe the EWSB improves for heavier tops, since (i) the QCD background decreases with increase in $m_{t}$, and (ii) the coupling of the top to the EWSB sector increases with $m_{t}$. In conclusion, we 
have demonstrated the feasibility of using $t \bar{t} t \bar{t}$ production as a probe to EWSB. Furthermore, we have shown that $m_{t \bar{t}}, \Delta p_{T}\left(t_{3}, t_{4}\right)$, and $y_{\text {gap }}$ serve as useful observables to identify the signal, especially for the colorless models. Further investigation is needed to distinguish the different underlying dynamics of the EWSB sector. This work was supported by the United States Department of Energy DE-FG03-93ER40757.

\section{References}

[1] F. Abe et al. (CDF Collaboration), FERMILAB-PUB-95-022-E, Mar 1995.

[2] S. Abachi et al. (D0 Collaboration), FERMILAB-PUB-95-028-E, Mar 1995.

[3] S. Weinberg, Phys. Rev. Lett. 19, 1264 (1967); A. Salam, in Elementary Particle Theory, edited by N. Svartholm (1968).

[4] M. Chanowitz and M. Gaillard, Nucl. Phys. B261, 379 (1985).

[5] see, e.g., D. Schaille, in Proceedings of the 1994 Int. Conf. on High Energy Physics, Glasgow, GB (1994).

[6] C. Hill and S. Parke, Phys. Rev. D50, (1994); D. Dicus and S. Nandi, Phys. Rev. D51, 6085 (1995).

[7] E. Eichten and K. Lane, Phys. Lett. B327, 129 (1994).

[8] C. Hill, Phys. Lett. B345, 483 (1995); E. Eichten and K. Lane, BUHEP-95-11, hepph/9503433 (1995).

[9] D. Dicus, A. Stange, and S. Willenbrock, Phys. Lett. B333, 126 (1994); C. Chiu and D.Dicus, Phys. Rev. D50, 6724 (1994).

[10] R.S. Chivukula and M. Golden, Phys. Rev. D41, 2795 (1990); R.S. Chivukula, in proceedings of 12th Johns Hopkins Workshop, Baltimore, MD (June 1988). 
[11] H.L. Lai et al. (CTEQ Coll.), Phys. Rev. D51,4763 (1995).

[12] V. Barger, A. Stange, and R.J.N. Phillips, Phys. Rev. D44, 1980 (1991).

[13] R.S. Fletcher and T. Stelzer, Phys. Rev. D48, 5162 (1993).

Table I: The effectiveness of the various cuts on the signal $g g \rightarrow t \bar{t} H \rightarrow t \bar{t} t \bar{t}$ and the QCD background $g g \rightarrow t \bar{t} t \bar{t}$.

\begin{tabular}{lcc}
\hline \hline Cuts & $g g \rightarrow t \bar{t} H \rightarrow t \bar{t} t \bar{t}$ & $g g \stackrel{\text { QCD }}{\rightarrow} \bar{t} t \bar{t}$ \\
\hline$(1)$ No cuts & $100 \%$ & $100 \%$ \\
$(2) p_{T}(t)>20 \mathrm{GeV},|y(t)|<5$ & $97.5 \%$ & $96 \%$ \\
$(3)$ cuts in (2) and $m_{t \bar{t}}>600 \mathrm{GeV}$ & $93 \%$ & $56 \%$ \\
$(4)$ cuts in (3) and $\Delta p_{T}\left(t_{3}, t_{4}\right)>600 \mathrm{GeV}$ & $73 \%$ & $23 \%$ \\
$(5)$ cuts in (4) and $\left|y_{\text {gap }}\right|>1.2$ & $49 \%$ & $10 \%$ \\
\hline
\end{tabular}

Figure Caption

1. Schematic diagram for the $t \bar{t}$ scattering in four-top production.

2. Normalized differential cross sections for the signal $g g \rightarrow t \bar{t} H \rightarrow t \bar{t} t \bar{t}$ and the QCD background $g g \rightarrow t \bar{t} t \bar{t}$ versus (a) $\Delta p_{T}\left(t_{3}, t_{4}\right)$ and (b) $y_{\text {gap }}=\left|y\left(t_{1}\right)-y\left(t_{2}\right)\right|$, after the cuts in Eqs. (14) and (16). 


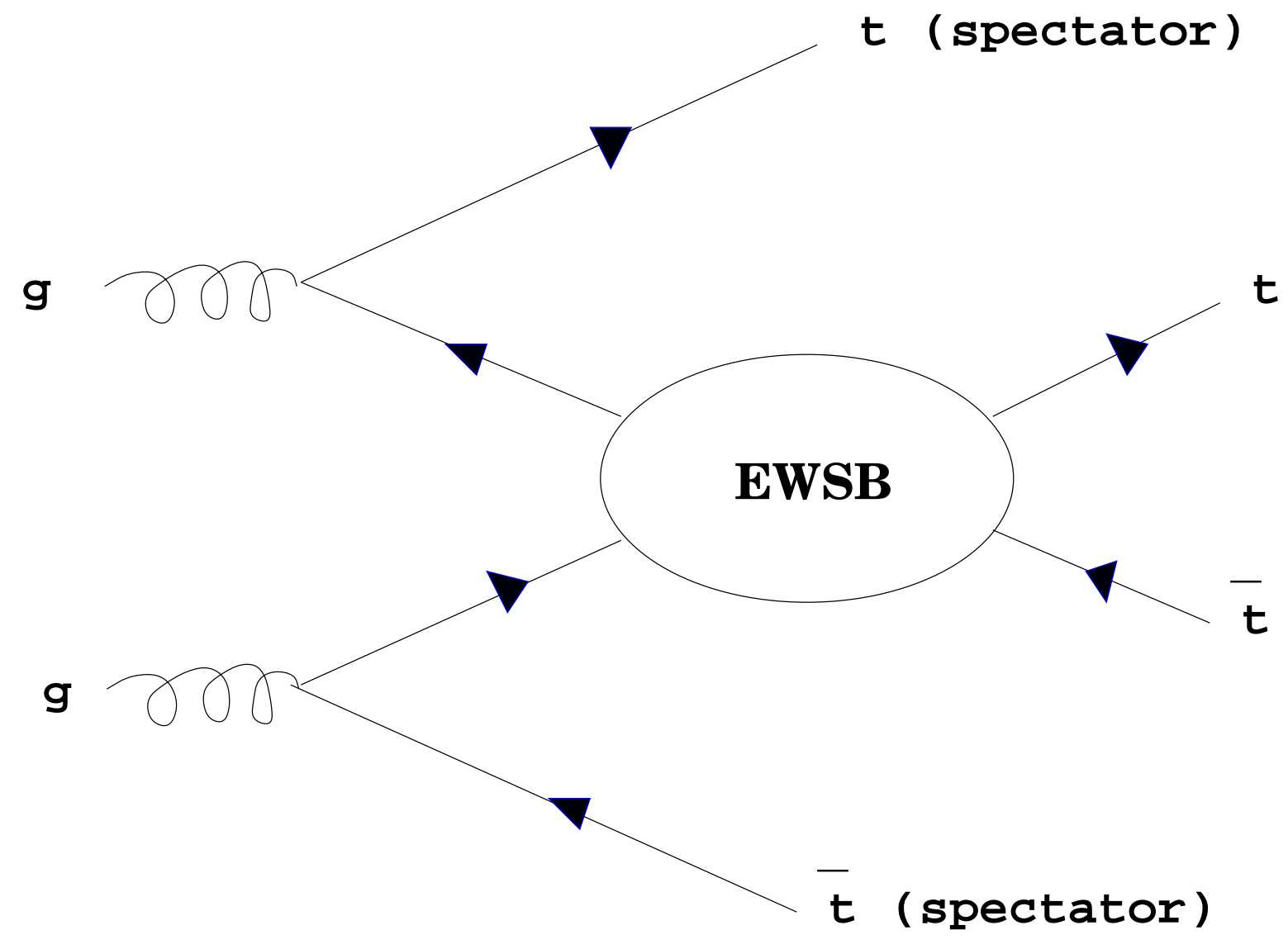




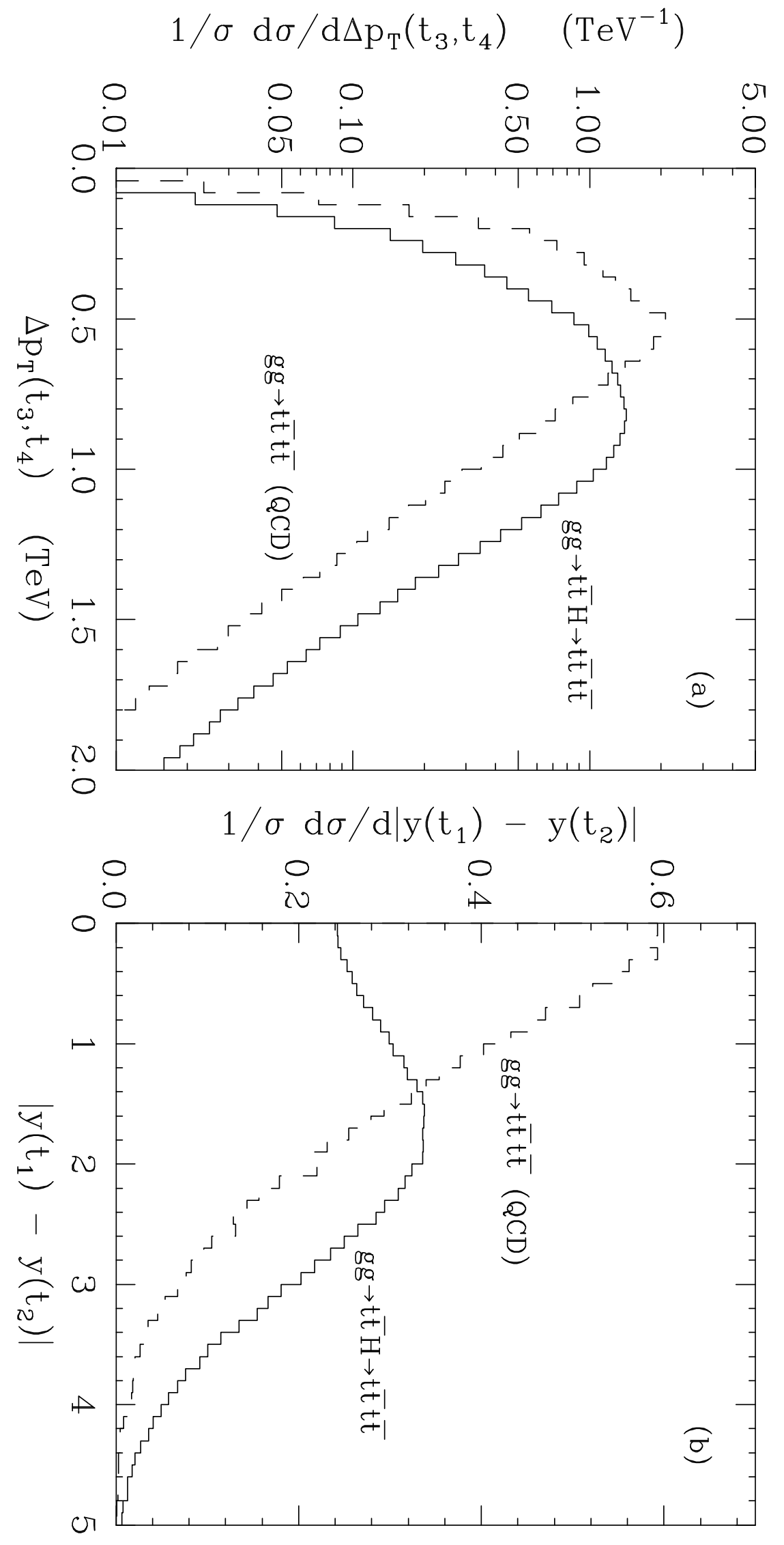

